\title{
A MULTIPLE ENCOUNTER MODEL OF M51
}

\author{
HEIKKI SALO and EIJA LAURIKAINEN \\ Division of Astronomy, Dept. of Physical Sciences, University of Oulu, Finland
}

The interaction of M51 with its companion NGC 5195 has been investigated with a spherical-polar grid code, where both components are described by self-gravitating star+gas disks embedded in analytical halo potentials. An extended survey was performed with various encounter geometries and companion masses, for relative orbits constrained by the components' observed separation and velocity difference. As analytical halos were used, these models describe only the current orbit. These calculations were thus complemented by live-halo experiments, in order to study the long-term orbital evolution due dynamical friction.

Two possible classes of current orbits were found: 1) nearly parabolic, single encounter orbits and 2) bound orbits implying several close passages. In both cases the major perturbation, capable to account for M51's prominent grand-design structure, took place when the companion crossed the disk plane of M51 about 500-600 Myrs ago, at distance of about 20-30 kpc. In the bound model this crossing was toward observer, and a second crossing occurred about 50-100 Myrs ago, enabling the companion to reach its present position and velocity.

Parabolic models have been previously presented by Hernquist (1990) and Toomre (1995), but inspite of their success in producing the main morphology of M51, these models are not totally satisfactory. To produce the extended HI-tail (Rots et al., 1990), either the encounter time was increased by about 2-3 times as compared to the classical study of Toomre and Toomre (1972), or the mass ratio was increased to unity. In the first case the extended tail was still slightly underdeveloped, whereas in the latter the mass of the companion is unrealistically large.

We have compared the two possible orbital classes in detail, with special attention on their kinematical implications. Both bound and parabolic models explain well the inner disk velocity field of M51. However, the observed high velocities to the north of the companion (up to $250 \mathrm{~km} \mathrm{~s}^{-1}$ relative to M51) can be induced only if a recent passage takes place, inducing a stream of gas from the main galaxy. Moreover, the bound model is able to account for the observed S-shaped major-axis rotation curve, eventhough its mass distribution remains close to initial one with nearly flat outer velocity curve. Most importantly, in the bound model the extended tail is tilted by about $40-50^{\circ}$ with respect to the inner disk, to the opposite side with respect to sky-plane. This agrees with the estimated tilt by Rots et al. (1990), based on apparent counter-rotating velocity field of the extended tail. 


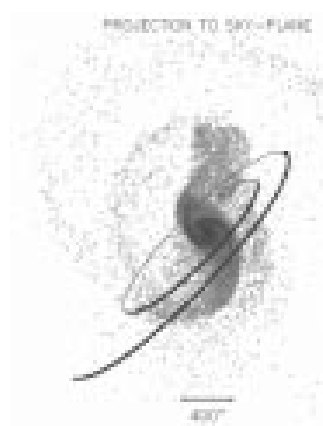

Figure 1. Example of multiple encounter model including live halo for M51. Companion disk is ignored while its total mass is 0.55 times the mass within M51's visible disk. Extended halo of M51 contains 2 times the visible mass.

Some morphological details are also better matched in the multiple encounter model. For example, the faint plumes associated to NGC 5195 show up in the observed east-west direction. Elmegreen (1989) first pointed out that the 'kink' in the northern arm of M51 is more pronounced than in the southern arm. This 'kink' in our simulations develops during the latest encounter. It is a place of vigorous star formation (Greenwalt et al., 1998), consistent with recent perturbation.

In live halo simulations the companion was followed through several disk plane crossings, taking place at successively shorter distances due to shrinking of the orbit by dynamical friction. Inspite of the long tidal history the present morphology of M51 with its most important observed kinematical properties is well matched (Figure 1), provided that the previous, more distant perturbations have been weak enough so that their effects have been overhelmed by the recent encounters.

\section{References}

Elmegreen, B.G., Elmegreen, D.M. and Seiden, P.E.: 1989, Astrophys. J. 343, 602-607.

Greenwalt, B., Walterbos, R.A.M., Thilker, D. and Hoopes, C.G.: 1998, Astrophys. J. 506, 135-151. Hernquist, L.: 1990, in: R. Wielen (ed.), Dynamics and Interactions of Galaxies, Heidelberg, Springer-Verlag, 108-117.

Rots, A.H., Bosma, A., van der Hulst, J.M., Athanassoula, E. and Crane, P.C.: 1990, Astron. J. 100, 387-393.

Toomre, A. and Toomre, J.: 1972, Astrophys. J. 178, 623-666.

Toomre, A.: 1995, Colloquim given at the Instituto de Astronomia, UNAM, April 1995. 